\title{
Poor diet quality in childhood cancer patients during treatment: A target for nutrition interventions
}

\author{
Jennifer Cohen ${ }^{1}$, Emma Goddard², Mary-Ellen Brierley ${ }^{1}$, Lynsey Bramley ${ }^{3}$, and Eleanor \\ Beck $^{2}$ \\ ${ }^{1}$ University of New South Wales Faculty of Medicine \\ ${ }^{2}$ University of Wollongong Illawarra Health and Medical Research Institute \\ ${ }^{3}$ Sydney Childrenś Hospital Randwick
}

July 16, 2020

\begin{abstract}
Background With improved survivorship and long-term health outcomes, the long-term nutritional management of childhood cancer survivors, from diagnosis to long-term follow-up, has become a priority. The aim of this study was to assess diet quality of children receiving treatment for cancer. Procedure Participants were parents of childhood cancer patients who were receiving active treatment and not receiving supplementary nutrition. A three-pass 24-hour dietary recall assessed food and nutrient intake. Serves of food group intakes and classification of core and discretionary items were made according to the Australian Dietary Guidelines and compared with age and sex recommendations. Results Sixty-four parents participated (75\% female). Nearly all children were not consuming adequate intake of vegetables (94\% of patients), fruit (77\%) and milk/alternatives (75\%). Of the vegetables that were consumed, half were classified as discretionary foods (e.g. chips/fries). Nearly half (49\%) of children exceeded recommendations for total sugar intake and $65 \%$ of patients had an excessive sodium intake. Discussion The diet quality of children undergoing treatment for cancer is generally poor. Information provided during treatment should focus on educating parents on a healthy diet for their child, the importance of establishing healthy eating habits for life, and strategies to overcome barriers to intake during treatment.
\end{abstract}

\section{Introduction}

The nutrition management of childhood cancer patients is an important aspect of their multidisciplinary care plan and medical management. The focus of this management has been on the prevention of undernutrition ${ }^{1}$. Without nutrition therapy, up to $50 \%$ of paediatric cancer patients are likely to become malnourished 2. Nutrition therapy in childhood cancer patients tends to focus on weight and growth-based outcomes whereby the maintenance of normal growth and development is the primary goal of nutrition interventions ${ }^{3}$. Algorithms for initiating nutrition supplementation are predominantly based on weight changes and the suggested interventions themselves rely on commercial supplements, enteral tube feeding and parenteral nutrition ${ }^{4}$.

Positively, recent research has shown that patients are meeting energy requirements during treatment ${ }^{5}$, as a consequence of a focus on symptoms affecting dietary intake. In children treated for childhood leukaemia, $80 \%$ of patients experience disrupted eating behaviour with issues such as nausea, changes in appetite, vomiting, food refusal and fussy eating affecting intake ${ }^{6-8}$. Consequently, parents often report changing their parenting strategies after diagnosis, including exerting higher levels of overprotectiveness, lower levels of discipline, offering nutrient-poor food rewards and non-food rewards for eating, and pressuring their child to eat ${ }^{9}$. There is a suggestion that the dietary intake of childhood cancer patients during treatment may be poor with research showing intake of poor quality foods and a reduction in food variety ${ }^{10,11}$. For those who 
are able to eat, food choices tend to be limited ${ }^{8}$ or patients have a preference for "junk food" ${ }^{12}$. It also appears that parents are not concerned about their child's overall diet quality during their cancer therapy ${ }^{13}$, as long as they are eating. Many childhood cancer patients are less than five years of age where long term feeding patterns and habits are being established ${ }^{14}$. In childhood cancer the triad of disease, its treatment, and feeding difficulties, may therefore lead to poor eating habits that persist long into adulthood.

As medical treatments have advanced, outcomes for childhood cancer patients have also improved significantly. In contrast to these obvious positive outcomes, chronic disease such as obesity and cardiovascular disease are being recognised as long term sequelae in adult survivors of childhood cancer ${ }^{15}$. Adult childhood cancer survivors have been shown to have poor dietary habits, with inadequate intake of fruit and vegetables, fibre and calcium and a high saturated fat intake ${ }^{16,17}$. It has also been shown that this poor dietary intake is manifesting within the early stages off treatment ${ }^{18,19}$. This is of particular concern as childhood cancer survivors have a predisposition to metabolic complications ${ }^{20}$ and there are high rates of obesity, especially in patients diagnosed with acute lymphoblastic leukaemia (ALL ${ }^{21}$. Although the documented poor dietary habits reflect current unhealthy eating habits across the broader population ${ }^{22}$, there is a need to address the dietary habits of childhood cancer patients during treatment.

A number of recent reviews highlighted the need for dietary intervention studies that aim to optimise therapy and survival, and reduce treatment-related late effects ${ }^{23,24}$. Decisions made regarding the nutrition management during cancer treatment the patient's diet quality and nutrient intake, may have the potential to influence short- and long-term morbidity and mortality ${ }^{25,26}$. There is a dearth of literature investigating the dietary intake of childhood cancer patients during treatment ${ }^{27}$. The aim of this study was therefore to assess the dietary intake and diet quality of children receiving treatment for cancer.

\section{Methods}

\section{Study Participants}

Participants were parents and/or primary carers of childhood cancer patients aged between two and 18 years receiving active treatment for cancer in a major metropolitan paediatric tertiary referral hospital. There were no exclusions based on cancer type although all children (of the parents) were required to be receiving active treatment. Where a child was undergoing chemotherapy but more than two weeks into the maintenance stage of their treatment regimen, parents/carers were excluded. The aim of the study was to assess the diet quality of childhood cancer patients who were relying on oral intake to maintain their nutritional status. Only children consuming food orally were included in the study, with those on enteral or parenteral feeds excluded. The participants were required to be sufficiently fluent in English to complete the written questionnaire.

\section{Recruitment}

Eligible participants were identified through hospital records by a qualified dietitian familiar with oncology patients at the hospital. All eligible participants were approached during outpatient clinic visits by a researcher not involved in the care of the participant's child. Potential participants were provided with information on the study, and invited to participate, with informed consent obtained in writing. Participants were recruited between April 2016 and February 2017. The study protocol was approved by the Sydney Children's Hospital Network ethics committee (EC00130).

\section{Data Collection}

Data was collected via a written questionnaire completed by the participant. The questionnaire was developed using both validated scales and items purposely designed by a team comprised of a dietitian, psychologist, clinical oncologist and psychosocial researcher. Sociodemographic questions of the participant covered age, sex, highest level of education, hours worked per week on average, self-reported weight and height, and relationship to the patient. Health literacy of participants was assessed using a validated four-item Brief Health Literacy Scale ${ }^{28}$. The four questions relate to an individual's ability to read and understand medical and hospital forms and level of understanding is assessed using a 5-point Likert scale. Scores range from 0-36. 
The socio-economic indexes for areas (SEIFA) index of relative socio-economic advantage and disadvantage was used to define participant socio-economic status by postal/zip code ${ }^{29}$. Scores range from one through to 10 , where 10 indicates high relative socio-economic advantage ${ }^{29}$.

Patient demographic characteristics collected included sex, date of birth, cancer type, stage and date of diagnosis, relapse status and treatment regimen. The information on the patient's diagnosis, stage, relapse status and treatment regimen were used to calculate the Intensity of Treatment Rating ${ }^{30}$. The Intensity of Treatment Rating provides four categories of treatment intensity ranging from level 1 which indicates the treatment is minimally invasive to level 4 which indicates the most invasive treatment ${ }^{30}$. Patient's current weight and height were also collected to calculate body mass index (BMI). BMI-for-age percentile and z-scores were adjusted for age and sex using the Centre for Disease Control and Prevention growth charts, to allow for comparisons across age groups. The BMI-for-age percentile was classified as either underweight $\left(<5^{\text {th }}\right.$ percentile), healthy weight $\left(5^{\text {th }}-84^{\text {th }}\right.$ percentile), overweight $\left(85^{\text {th }}-94^{\text {th }}\right.$ percentile $)$ or obese $\left(>95^{\text {th }} \text { percentile }\right)^{31}$.

One day of dietary intake was assessed using a three-pass 24 hour dietary recall ${ }^{32}$. This method consists of a structured interview conducted by the study coordinator who asks the adult caregiver to list everything the child ate or drank during the previous day. The three-pass 24-hour dietary recall is considered to have one of the highest validation standards for dietary assessment methods as the method utilises a very structured interview procedure that includes three distinct probing sessions (or passes) during the interview.

32 The 10-item Peds-FAACT:Pediatric - Functional Assessment of Anorexia Cachexia sub-scale was used as a subjective measure of the severity of food related symptoms such as taste change and poor appetite ${ }^{33}$. Questions include subjective views of symptoms such as early satiety, nausea, vomiting and taste changes in the previous seven days. A 5 -point Likert scale $(0=$ not at all to $5=$ very much) was used to score each question.

\section{Data Analysis}

Dietary data from the 24-hour recalls was analysed using the FoodWorks nutrient analysis software program (version 8, 2015; Xyris Software, Queensland, Australia). For food and drink items missing from the FoodWorks database, nutrient content was obtained from product nutrition panels and entered manually. Serves of food group intake (fruits, vegetables, grains, meat/alternatives and milk/alternatives) were automatically calculated by FoodWorks dietary analysis software (Xyris software, Version 8). Foods were then classified as core or discretionary foods by a researcher according to the 2013 Australian Dietary Guidelines ${ }^{34}$ whereby discretionary foods are described as higher in energy density, saturated fat, sodium, sugars and or alcohol). Manual extractions of discretionary foods were required in some instances. For example, fruit juice is considered a discretionary item so was separated from fruit intake. Food intake data was compared to age and sex appropriate recommended serves for Australian children ${ }^{34}$. Dietary intake results for children in the general Australian population and were obtained from the 2011-12 Australian Health Survey ${ }^{35,36}$ and used as a comparative norm. Food group intake was expressed as a percent of serves recommended by the Australian Dietary Guidelines ${ }^{34}$ to allow for comparisons between age groups.

A nutrient was included for dietary assessment if an appropriate Nutrient Reference Value or Australian Dietary Guideline recommendation existed. Incorporating all nutrients possible contributes to a thorough assessment of dietary quality. Children's nutrient intake was expressed as a percentage of their age and sex appropriate estimated average requirement (EAR) or adequate intake (AI) when EAR was not available ${ }^{37}$. The estimated energy requirement of each child was calculated using age appropriate Schofield equations ${ }^{38}$ multiplied by a physical activity level of 1.5 (sedentary) ${ }^{39}$. The Schofield equation calculates an estimation of a person's basal metabolic rate based on their age, gender and weight ${ }^{40}$. Total energy intake was expressed as a percentage of estimated energy intake (\%EER) for each child which allowed mean \%EER to be calculated. Intake of carbohydrate, protein and fat was also expressed as a \%EER to determine macronutrient distribution ranges and compare these to the acceptable macronutrient distribution ranges for reduced risk of chronic disease ${ }^{34}$. Descriptive statistics for demographic and dietary intake data were calculated using SPSS statistics (version 21.0, 2012; IBM Corp., Armonk, NY). 
Assumptions of normality did not appear to hold for some variables, therefore for consistency non-parametric tests were used. Non-parametric tests (Mann-Whitney and Kruskall-Wallis) were used to compare the difference in dietary intake between patient sex (male versus female), diagnosis (ALL versus other) and treatment intensity. As children treated for ALL are at a higher risk of obesity than other diagnoses ${ }^{21}$, a comparison of the dietary intake between children with ALL and other diagnoses was undertaken. Statistical significance was set at a level of 0.05 with no adjustment for multiple comparisons.

\section{Results}

\section{Participant Demographics}

Sixty-eight participants were approached and invited to participate in this study. Surveys were distributed to 67 parents/carers of paediatric cancer patients. Sixty-four participants completed the 24-hour recall and questionnaire representing a $94 \%$ response rate. Of the surveys returned, three were excluded from the analysis as one was a duplicate, one paediatric patient was on maintenance treatment, and one child had turned 18 during the survey and did not provide their own consent, resulting in a sample size of sixty-one. Demographic information (Table 1) showed mean age (SD) of the childhood cancer patients was $8( \pm 4.47)$ years. The majority children $(68 \%)$ were a healthy weight for their height and $3 \%, 12 \%$ and $14 \%$ were considered underweight, overweight and obese respectively.

\section{Dietary Intake}

Dietary data analysis revealed $50 \%$ of children were exceeding their \%EER by greater than $10 \%$, although $19 \%$ were consuming less than $75 \%$ of their \%EER. Patients were consuming excessive protein intake with a mean intake of $400 \%$ of requirements and only $5 \%$ not meeting their protein requirements. The patients mean nutrient intake all exceeded EARs indicating generally, that it is unlikely individuals are deficient in specific nutrients. The percentage of children not meeting the EAR/AI was lower than the normative data from the Australian Health Survey (except for magnesium and calcium), however, using the crude measure of EAR it cannot be determined if any children were at risk of deficiency. In contrast, when dietary quality was considered, particularly with respect to nutrients associated with increased risk of chronic disease, large numbers of children exceeded recommendations. Sugar intake was excessive, with nearly half $(49 \%)$ of children exceeding recommendations for total sugar intake ( $>20 \%$ total energy from sugars) and $61 \%$ exceeding recommendations for added sugar intake ( $>10 \%$ total energy from added sugars). More than half of the children were not meeting their AI recommendations for fibre (61\%). Sodium intake was calculated as excessive (intake $>100 \%$ of the highest bound range of AI) in $65 \%$ of children, although this percentage was slightly lower than normative data (Table 2).

There was no significant difference between intakes of core food groups between male and female or between patients with ALL and other diagnoses (Supplementary Table 1). There were no significant differences in intake for any food groups and treatment intensity except for core grain foods $(\mathrm{p}=0.017)$, with core grain intake greater amongst those on moderate intensity treatment compared to all other intensities.

Considering food group data as a further measure of dietary quality, many children did not meet minimum serves recommended for intake of all core food groups (Table 3). It is important to consider the intake excluding discretionary foods, as these foods add excessive saturated fat, sodium and sugar, and insufficient dietary fibre and whole grains. The intake of core foods was like the Australian Health Survey normative data. When this data was reviewed to include intake from discretionary items, the general lack of dietary nutrient deficiency (Table 2) was explained. Patients had a larger intake from most food groups compared to normative data, however the discretionary nature of their intake indicated poorer dietary quality. Half (51\%) of total vegetable intake serves were classified as discretionary according to the Australian Dietary Guidelines. Foods contributing significantly to discretionary vegetable intake included takeaway fries, savoury chips/crisps and tomato sauce.

Fruit juice contributed to $47 \%$ of total fruit intake. Core foods contributed to greater than $65 \%$ of food group 
intake for grains, meat and dairy. Discretionary food items contributing to total grain food group intake included pastry products, baked goods and sweet and savoury biscuits. Refined grains, if from core foods (e.g. breads, cereals) are still classified as core foods. However, whilst grains classified as discretionary foods contributed to only $31 \%$ of grain intake, refined grains contributed to $71 \%$ of grain intake. The Australian Dietary Guidelines recommend grain consumption to consist mostly of whole grains. Core foods contributing to refined grain intake included white bread, rice and pasta. Foods contributing to meat and milk alternatives intake were mostly classified as core foods, though there were contribution from non-core foods, with some amounts of processed meats including bacon and salami contributing to meat intake, and small amounts of ice cream and chocolate contributing to dairy.

\section{Food Related Symptoms}

The most common symptoms parents reported their children were experiencing were taste changes $(17.8 \%)$ and early satiety (14.6\%) (Figure 1). Almost $60 \%$ of parents reported that their child was able to "eat as much as they wanted" and only $4 \%$ of carers reported needing to pressure their child to eat.

\section{Discussion}

This study aimed to describe the dietary intake and dietary quality of children during their treatment for cancer. The dietary data collected in this study suggests that children receiving cancer treatment are consuming diets of reasonable quantity, but poor quality. There were some potential inadequacies of nutrients, and under-representation of core food groups were seen amongst most of this childhood cancer population. This study also found that many parents reported that their children were able to eat what they liked and did not experience issues with treatment related side effects such as nausea or vomiting.

Overweight and obesity and its consequent inflammation is linked with the sequelae of metabolic diseases ${ }^{41}$ of particular concern in childhood cancer survivors as rates of these disease are higher in this group ${ }^{15}$. Our study found half of the children being treated for cancer were consuming greater than $110 \%$ of their energy requirements. It appears that for some patients, the excessive energy intake seen at the end of treatment is manifesting during treatment ${ }^{19}$. Excessive energy intakes resulting in weight gain places a childhood cancer survivor at an even higher risk of developing a chronic health condition post treatment completion 42. A recent study has shown that it is possible to prevent excessive weight gain with a targeted nutrition intervention during maintenance therapy ${ }^{43}$ though it may be that targeted interventions need to occur during early cancer treatment ${ }^{23}$.

Comparisons to normative data from the Australian Health Survey suggest that fruit and vegetable intake of childhood cancer patients was greater than that of Australian children in the general population, primarily related to intake of discretionary foods, with fruit juice contributing to more than half of total fruit intake. Vegetable based discretionary foods including takeaway fries and chips comprised the majority of discretionary vegetable intake suggesting that total vegetable intake was of a poor nutrient quality. Given the role that adequate food intake of core vegetables, fruit and whole grains play in prevention of chronic disease ${ }^{44}$, assisting families to improve diet quality in these food groups is important. Additionally, consuming a nutrient rich diet comprised of fruits, vegetables and whole grains may contribute to long-term weight maintenance among survivors of childhood cancer ${ }^{45}$.

The goal of both parents and clinicians working in paediatric oncology until now has been the prevention of weight loss through the use of a high energy diet ${ }^{46}$. This may encourage an 'anything goes' approach to intake, particularly intake of high energy foods. (11) Many nutrition information resources encouraging consumption of discretionary foods, including those high in saturated fat such as ice-cream, hot chips and fortification with butter and cream. Given that these poor dietary habits established during cancer treatment may persist into adulthood (3), a shift in focus from merely weight and weight maintenance is required. Specifically, clinical teams need to assist in establishment of good dietary habits, while managing side effects and barriers to achieving adequate intake. It may also be that dietary advice during cancer treatment may need to be targeted depending on treatment intensity and the potential for nutrition-related side effects. Focussing on dietary quality ${ }^{23}$ while still avoiding malnutrition, both under- and over-nutrition ${ }^{15,20}$, is required. 
There will still be some patients who receive high intensity treatment and are therefore known to have a high nutrition risk disease. This increases their likelihood of requiring supplementary nutrition, and a continued focus on the prevention of weight loss is required. However, for other paediatric oncology patients the nutrition therapy may need to be altered. Specifically, this may require a shift away from monitoring weight only and instead reviewing the nutritional value of the whole diet, working with families to manage food related treatment symptoms, and establishing long-term healthy dietary patterns to avoid risks to longterm health ${ }^{15,20}$. Barriers to intake such as fussy or picking eating, are often not unique to sick children (27). In fact, one study found very few differences in eating and mealtime behaviours between children receiving cancer treatment and healthy children (27). This suggests that nutrition information for parents of children with cancer should not necessarily be dissimilar to regular messages provided to parents including eating a healthy, balanced diet, establishing good eating habits, and consuming fruits, vegetables, and wholegrains.

\section{Limitations}

The dietary data should be interpreted with caution as dietary intake information was collected crosssectionally using a 1-day 24-hour diet recall and may not be indicative of the larger picture of the changing dietary quality during treatment. Future studies should consider the use of the 24-hour diet recall assess over a three-day period to assess dietary intake ${ }^{47}$ with dietary intake assessed longitudinally throughout cancer treatment. This study had a small sample size which resulted in an uneven sample distribution across age groups. Therefore, identification of groups most at risk of poor dietary quality could not be concluded. Additionally, the sample was heterogeneous across cancer diagnosis and treatment, making it difficult to draw conclusions regarding dietary quality across treatment intensity. The study did not adjust for multiple comparisons with the possibility of an inflated Type I error. Due to this, the results need to be interpreted with caution. Nutrition information needs will vary for different cancer and treatment types; for example, steroid use will drive up hunger ${ }^{21}$ whereas other treatments can cause nausea, vomiting and other symptoms that impact intake. The study excluded patients who were receiving enteral nutrition during the study period. Although childhood cancer patients receiving enteral nutrition rely on supplementary feeding for their nutritional intake, some patients may also be consuming some oral intake. Excluding these patients may have introduced some sampling bias and future studies should aim to assess the dietary intake of all patients during active treatment. High levels of health literacy and socio-economic status among study participants may have influenced the results of this study. The results from this study may not be transferrable to other oncology populations, especially those from developing countries where treatment and dietary advice may vary.

\section{Conclusion}

The current study shows that diet quality of children undergoing treatment for cancer is generally poor. Given the high survivorship rates and increased risk of chronic disease, information provided during treatment should focus on educating parents on a healthy diet for their child, the importance of establishing healthy eating habits for life, and strategies to overcome barriers to intake during treatment. We no longer need to use nutrition therapy as a "one-size-fits-all" approach but should be based on treatment protocols and their nutrition risk. There needs to be further longitudinal research assessing change in dietary intake throughout treatment. There is also a need for greater research on whether the dietary quality of patients can be improved during cancer therapy with targeted interventions. Future research is also required to determine whether specific dietary patterns improve survival both during and after treatment.

\section{Conflicts of interest}

The authors declare that they have no conflict of interest

\section{References}

1. Coates TD, Rickard KA, Grosfeld JL, Weetman RM. Nutritional support of children with neoplastic diseases. Surgical Clinics of North America. 1986;66(6):1197-1212.

2. Brinksma A, Huizinga G, Sulkers E, Kamps W, Roodbol P, Tissing W. Malnutrition in child- 
hood cancer patients: a review on its prevalence and possible causes. Critical Reviews in OncologyHematology.2012;83(2):249-275.

3. Ladas EJ, Sacks N, Meacham L, et al. A multidisciplinary review of nutrition considerations in the pediatric oncology population: a perspective from children's oncology group. Nutrition in Clinical Practice. 2005;20(4):377-393.

4. Bowman LC, Williams R, Sanders M, Ringwald-Smith K, Baker D, Gajjar A. Algorithm for nutritional support: Experience of the Metabolic and Infusion Support Service of St. Jude children's Research Hospital.International Journal of Cancer. 1998:76-80.

5. Brinksma A, Roodbol PF, Sulkers E, et al. Finding the right balance: An evaluation of the adequacy of energy and protein intake in childhood cancer patients. Clinical nutrition. 2015;34(2):284-290.

6. Green R, Horn H, Erickson JM. Eating experiences of children and adolescents with chemotherapy-related nausea and mucositis. J Pediatr Oncol Nurs. 2010;27(4):209-216.

7. Hanigan MJ, Walter GA. Nutritional support of the child with cancer.J Pediatr Oncol Nurs. 1992;9(3):110118.

8. Skolin I, Hursti U-KK, Wahlin YB. Parents' perception of their child's food intake after the start of chemotherapy. J Pediatr Oncol Nurs. 2001;18(3):124-136.

9. Fleming CAK, Cohen J, Murphy A, Wakefield CE, Cohn RJ, Naumann FL. Parent feeding interactions and practices during childhood cancer treatment. A qualitative investigation. Appetite. 2015.

10. Cohen J, Wakefield CE, Tapsell LC, Walton K, Fleming CA, Cohn RJ. Exploring the views of parents regarding dietary habits of their young cancer-surviving children. Supportive Care in Cancer.2015;23(2):463471.

11. Malihi Z, Kandiah M, Chan Y, et al. The effect of dietary intake changes on nutritional status in acute leukaemia patients after first induction chemotherapy. European journal of cancer care.2015;24(4):542-552.

12. Rickard KA, Grosfeld JL, Coates TD, Weetman R, Baehner RL. Advances in nutrition care of children with neoplastic diseases: a review of treatment, research, and application. Journal of the American Dietetic Association. 1986;86(12):1666-1676.

13. Gerhardt CA, Baughcum AE, Johnston A, et al. Parent perceptions of nutritional issues during their child's treatment for cancer.Journal of Pediatric Hematology/Oncology. 2006;28(7):454-460.

14. Northstone K, Emmett PM. Are dietary patterns stable throughout early and mid-childhood? A birth cohort study. British journal of nutrition. 2008;100(5):1069-1076.

15. Oeffinger KC, Mertens AC, Sklar CA, et al. Chronic health conditions in adult survivors of childhood cancer. New England Journal of Medicine. 2006;355(15):1572-1582.

16. Demark-Wahnefried W, Werner C, Clipp EC, et al. Survivors of childhood cancer and their guardians. Cancer.2005;103(10):2171-2180.

17. Belle FN, Chatelan A, Kasteler R, et al. Dietary intake and diet quality of Swiss adult survivors of childhood cancer compared to the general population. bioRxiv. 2019:527424.

18. Cohen J, Wakefield. C.W, Fleming, C.A.K, Cohn, R.J. Consequences of treatment on the food prefences and dietary habits of childhood cancer survivors. 43 Annual Conference of the International Society of Paediatric Oncology; Nov 2011, 2011 Aukland New Zeland.

19. Cohen J, Wakefield CE, Fleming CAK, Gawthorne R, Tapsell LC, Cohn RJ. Dietary intake after treatment in child cancer survivors. Pediatric Blood $\mathcal{E}$ Cancer. 2012;58(5):752-757. 
20. Diller L, Chow EJ, Gurney JG, et al. Chronic disease in the Childhood Cancer Survivor Study cohort: a review of published findings. Journal of Clinical Oncology. 2009;27(14):2339-2355.

21. Touyz LM, Cohen J, Neville KA, et al. Changes in body mass index in long-term survivors of childhood acute lymphoblastic leukemia treated without cranial radiation and with reduced glucocorticoid therapy.Pediatric blood $\&$ cancer. 2017;64(4):e26344.

22. Stolley MR, Sharp LK, Arroyo C, Ruffin C, Restrepo J, Campbell R. Design and recruitment of the Chicago Healthy Living Study: a study of health behaviors in a diverse cohort of adult childhood cancer survivors. Cancer. 2009;115(18 Suppl):4385-4396.

23. Schadler KL, Kleinerman ES, Chandra J. Diet and exercise interventions for pediatric cancer patients during therapy: tipping the scales for better outcomes. Pediatric research. 2018;83(1-1):50.

24. Wallace TC, Bultman S, D'Adamo C, et al. Personalized Nutrition in Disrupting Cancer-Proceedings From the 2017 American College of Nutrition Annual Meeting. Journal of the American College of Nutrition. 2018:1-14.

25. Kanarek N, Petrova B, Sabatini DM. Dietary modifications for enhanced cancer therapy. Nature. 2020;579(7800):507-517.

26. Ladas EJ, Blonquist TM, Puligandla M, et al. Protective Effects of Dietary Intake of Antioxidants and Treatment-Related Toxicity in Childhood Leukemia: A Report From the DALLT Cohort. Journal of Clinical Oncology. 2020:JCO. 19.02555.

27. Goddard E, Cohen J, Bramley L, Wakefield CE, Beck EJ. Dietary intake and diet quality in children receiving treatment for cancer.Nutrition reviews. 2019;77(5):267-277.

28. Haun J, Noland-Dodd V, Varnes J, Graham-Pole J, Rienzo B, Donaldson P. Testing the BRIEF health literacy screening tool. Federal Practitioner. 2009;26(12):24-31.

29. Australian Bureau of Statistics. Information Paper: An Introduction to Socio-Economic Indexes for Areas (SEIFA), 2006. In. Canberra: Australian Bureau of Statistics; 2008.

30. Kazak AE, Hocking MC, Ittenbach RF, et al. A revision of the intensity of treatment rating scale: classifying the intensity of pediatric cancer treatment. Pediatric blood \& cancer.2012;59(1):96-99.

31. Daniels SR. The use of BMI in the clinical setting.Pediatrics. 2009;1(124):S35-S41.

32. McPherson RS, Hoelscher DM, Alexander M, Scanlon KS, Serdula MK. Dietary Assessment Methods among School-Aged Children: Validity and Reliability. Preventive Medicine. 2000;31(2):S11-S33.

33. Lai J-S, Cella D, Kupst MJ, et al. Measuring fatigue for children with cancer: development and validation of the pediatric Functional Assessment of Chronic Illness Therapy-Fatigue (pedsFACIT-F).Journal of Pediatric Hematology/Oncology. 2007;29(7):471-479.

34. National Health and Medical Research Council. Australian Dietary Guidelines. In. Canberra: Australian Government, Department of Health and Ageing; 2013.

35. Australian Bureau of Statistics. Australian Health Survey:

Usual Nutrient Intakes, 2011-12. cat. no. 4364.0.55.008. http://www.abs.gov.au/ausstats/abs@.nsf/Lookup/by\%20Subject/4364.0.55.008 2011-

$12^{\sim}$ Main\%20Features ${ }^{2}$ Preface $\sim 1$.

36. Australian Bureau of Statistics. Australian Health Survey: Consumption of Food Groups from the Australian Dietary Guidelines, 2011-12. cat. no. 4364.0.55.012. 2016; $\quad$ http://www.abs.gov.au/ausstats/abs@.nsf/Lookup/by\%20Subject/4364.0.55.012 2011$12^{\sim}$ Main\%20Features $\sim$ Key\%20Findings $\sim 1$. 
37. National Health and Medical Research Council. Nutrient reference values for Australia and New Zealand including recommended dietary intakes. In. Canberra: Australian Government, Department of Health and Ageing; 2005.

38. Schofield WN. Predicting basal metabolic rate, new standards and review of previous work. Human Nutrition Clinical Nutrition.1985(S39):5-41.

39. Aznar S, Webster AL, San Juan AF, et al. Physical activity during treatment in children with leukemia: a pilot study. Applied Physiology, Nutrition 8 Metabolism. 2006;31(4):407-413.

40. Schofield W. Predicting basal metabolic rate, new standards and review of previous work. Human nutrition Clinical nutrition.1985;39:5-41.

41. Singer K, Lumeng CN. The initiation of metabolic inflammation in childhood obesity. The Journal of clinical investigation.2017;127(1):65-73.

42. Fuemmeler BF, Pendzich MK, Clark K, et al. Diet, physical activity, and body composition changes during the first year of treatment for childhood acute leukemia and lymphoma. Journal of pediatric hematology/oncology. 2013;35(6):437.

43. Hill R, Hamby T, Bashore L, et al. Early nutrition intervention attenuates weight gain for pediatric acute lymphoblastic leukemia patients in maintenance therapy. Journal of pediatric hematology/oncology. 2018;40(2):104-110.

44. Yoo S, Nicklas T, Baranowski T, et al. Comparison of dietary intakes associated with metabolic syndrome risk factors in young adults: the Bogalusa Heart Study. The American journal of clinical nutrition.2004;80(4):841-848.

45. Smith WA, Li C, Nottage KA, et al. Lifestyle and metabolic syndrome in adult survivors of childhood cancer: a report from the St. Jude Lifetime Cohort Study. Cancer. 2014;120(17):2742-2750.

46. Mauer AM, Burgess JB, Donaldson SS, et al. Special nutritional needs of children with malignancies: a review. Jpen: Journal of Parenteral \& Enteral Nutrition. 1990;14(3):315-324.

47. Burrows TL, Martin RJ, Collins CE. A systematic review of the validity of dietary assessment methods in children when compared with the method of doubly labeled water. Journal of the American Dietetic Association. 2010;110(10):1501-1510.

\section{Figure Legend}

Food-related symptoms experienced during treatment

\section{Hosted file}

Table 1.docx available at https://authorea.com/users/343437/articles/470074-poordiet-quality-in-childhood-cancer-patients-during-treatment-a-target-for-nutritioninterventions

\section{Hosted file}

Table 2.docx available at https://authorea.com/users/343437/articles/470074-poordiet-quality-in-childhood-cancer-patients-during-treatment-a-target-for-nutritioninterventions

\section{Hosted file}

Table 3.docx available at https://authorea.com/users/343437/articles/470074-poordiet-quality-in-childhood-cancer-patients-during-treatment-a-target-for-nutritioninterventions 


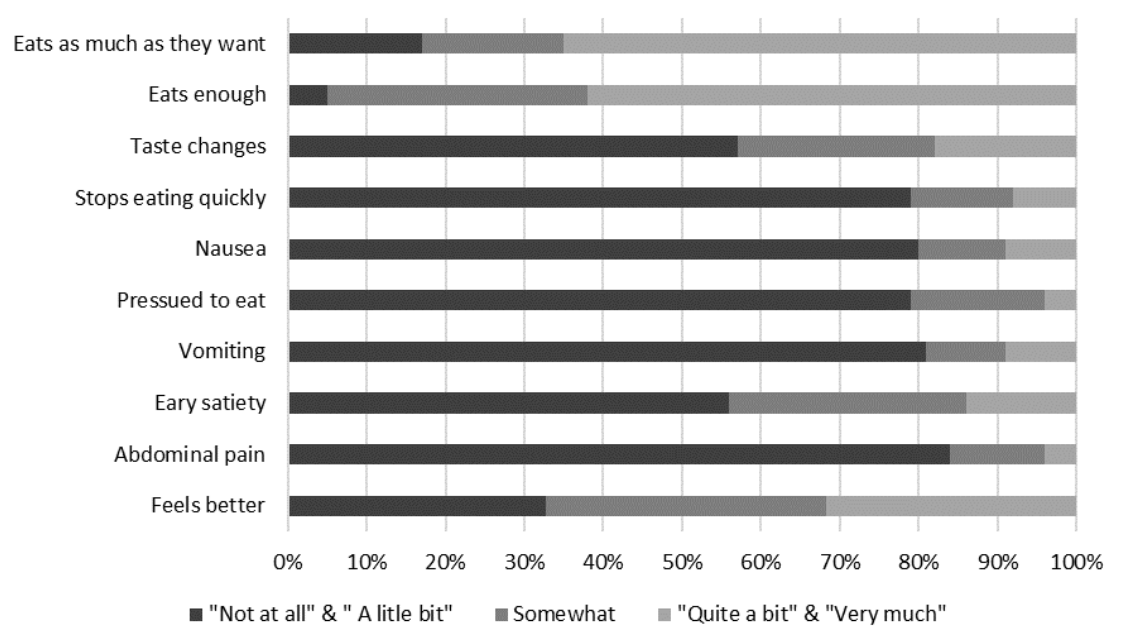

